\title{
Roughness Measurements with Polychromatic Speckles on Tilted Surfaces
}

\author{
Johannes Stempin ${ }^{1}$ (1) $\cdot$ Andreas Tausendfreund ${ }^{1} \cdot$ Dirk Stöbener $^{1} \cdot$ Andreas Fischer $^{1}$
}

Received: 14 September 2020 / Revised: 4 December 2020 / Accepted: 17 December 2020 / Published online: 1 March 2021

(c) The Author(s) 2021

\begin{abstract}
Surface light scattering enables contactless and fast measurements of surface roughness. A surface inclination alters the direction of the scattering beam and thus the measured surface roughness is calculated from the detected intensity distribution. Hence, an accurate sensor-surface alignment is necessary. In order to achieve tilt-independent roughness measurements, a model-based evaluation approach for polychromatic speckle patterns is presented. By evaluating the shape of the superposed speckles, which occur for polychromatic illumination, with regard to the distance to the scattering centre, surfaces with an $\mathrm{Sa}$ roughness value in the range of $0.8-3.2 \mu \mathrm{m}$ are measurable. Experimental investigations demonstrate that the influence of a surface tilt up to $\pm 1.25^{\circ}$ on the roughness measurement is reduced by $90 \%$. As a result, the robustness of the polychromatic speckle roughness measurement is improved, which allows to speed up the adjustment of the measurement system or the surface sample, respectively.
\end{abstract}

Keywords Surface roughness $\cdot$ Polychromatic light scattering $\cdot$ Surface tilting $\cdot$ In-process measurement

\section{Introduction}

Roughness is one important surface characteristic that is used to evaluate the quality of manufactured components. A usual roughness parameter is the arithmetic mean roughness

$S a=\frac{1}{A} \cdot \iint_{A}|z(x, y)| \mathrm{d} x \mathrm{~d} y$.

The recording of surface roughness in process chains is usually performed at the chains end by random sampling. This increases the manufacturing lead time and the surface quality cannot be guaranteed for all components with justifiable effort. For in-line measurements, a fast roughness measurement principle is required and a known surface tilt with respect to the sensor axis cannot be assured. A suitable measurement system should therefore enable fast, preferably areal measurements of the surface roughness for an unknown surface tilt.

Johannes Stempin

j.stempin@bimaq.de

1 Bremen Institute for Metrology, Automation and Quality Science, University of Bremen, Linzer Str. 13, 28359 Bremen, Germany
A standard measurement technique for the surface roughness is using tactile probes. Tactile methods detect the roughness by evaluating the deflections of a stylus that moves over the surface. Although high precision and a sufficiently large measurement range are achievable, the pointwise scanning of large areas is too slow for process-internal measurements [1].

Optical methods operate contactlessly and in principle provide the fastest possible speed (light speed). As an example, white light interferometry allows a precise acquisition of the surface height information. Due to the scanning operating principle, however, a fast roughness measurement of large areas is still challenging. Recent studies concerning the process-internal usage also show that a tilting of the measuring surface leads to an additional measurement uncertainty of approximately $0.2 \mu \mathrm{m} /$ degree [2].

Instantaneous unidimensional and areal measurements of the surface roughness can be performed with scattered light methods. For instance, total integrated scattering analyzes the relation of the incident beam intensity to the intensity of the scattered light [3]. However, it requires a complex measurement setup, which impedes the use in the production chain. Commercially available measuring devices are based on an angle-resolved scattering analysis. They are capable of roughness and shape measurements of 
components in production within a roughness measuring range of $0.01 \mu \mathrm{m}<S a<2 \mu \mathrm{m}$ at measurement frequencies of $2 \mathrm{kHz}$ [4]. The standard measuring spot size is $0.9 \mathrm{~mm}$ at a measuring distance of $5 \mathrm{~mm}$ and surface tilts within $\pm 5.7^{\circ}$ are evaluable. However, they are only capable of acquiring surface roughness information along a measurement line. Hence, the direction of interest on the surface must be known and thoroughly aligned to the device.

An alternative approach is to use coherent light. If a rough surface is illuminated with coherent light, constructive and destructive interference produces a granular intensity distribution in the plane of observation, so-called speckle [5]. Speckles form the basis for various measurement techniques, which can be used to detect deformations or highly accurate changes in distance [6]. Also, the surface roughness information contained in the speckle pattern is extractable by different speckle correlation techniques. The monochromatic speckle correlation evaluates the autocorrelation function of a captured speckle pattern [7]. The technique is applicable for smooth surfaces with $\mathrm{Sa}<\frac{\lambda}{4}$ [8]. Angular speckle correlation uses the correlation of speckle patterns obtained from two different angles of illumination [9]. This technique requires a knowledge of the illumination angles and thus also the surface tilt [10]. A slightly varied wavelength results in a similar but not identical speckle pattern that is stretched in the radial direction from the scattering centre. Hence, for polychromatic illumination with close wavelengths but identical angles of illumination, the speckles overlap in the image recorded with a monochrome camera and form radially symmetrical, linear structures with respect to the scattering centre [11]. This phenomenon is called speckle elongation, and it has been shown that the elongation effect depends on the surface roughness $[12,13]$. The elongation, respectively, the roughness can be evaluated by the use of the autocorrelation function [14]. Polychromatic speckle correlation enables roughness measurements in a range of $0.05 \mu \mathrm{m}<S a<5 \mu \mathrm{m}$, for instance with a measuring spot size of $1 \mathrm{~mm}$ at a measuring distance of $100 \mathrm{~mm}$ [14]. A non-zero tilt angle disturbs the measurement and theoretical studies on the effects of a surface tilt were performed in [15]. However, a measurement approach that is insensitive with respect to a surface tilt over a wide tilt angle range is missing.

For this reason, the aim of the article is to introduce a model-based measuring principle that evaluates polychromatic speckle patterns and enables measuring the roughness parameter $S a$ independently of the surface tilt. The measurement principle that is derived according to the polychromatic speckle theory from [16] is presented in Sect. 2. In Sect. 3 the experimental setup to validate the measurement principle is presented, and in Sect. 4 experimental results are discussed. A summary and outlook of the work on future research activities follow in Sect. 5.

\section{Measurement Principle}

Figure 1 shows the measurement arrangement using polychromatic speckle patterns. The surface to be examined is illuminated with a polychromatic laser beam, while the measuring spot corresponds to the beam diameter on the surface. The scattered light is focused via a biconvex lens onto a monochrome camera, which is placed at a distance equal to the focal length $f$ of the biconvex lens. Figure 2 a shows an example of a polychromatic speckle pattern that is captured with the monochrome camera over the camera coordinates $\xi$ and $\eta$. Note that the origin of the coordinates $\xi, \eta$ is set to the scattering centre $\left(\xi_{0}, \eta_{0}\right)$, where the speckles have a round shape. The radial elongation of the superposed polychromatic speckles spreads from the scattering centre, i.e., from $(0,0)$. The position of the scattering centre in Fig. $2 \mathrm{a}$ is equal to the position of the optical axis, since the surface here is perpendicular to the optical axis. With increasing distance from the scattering centre the radial speckle elongation at first increases, since the speckles of the individual wavelengths have an increasing distance to each other. The polychromatic speckle elongations $\Delta \xi_{s p}, \Delta \eta_{s p}$ in $\xi$ - and $\eta$ -direction, respectively, are defined by the $1 / \mathrm{e}$ decrease of the 2D-autocorrelation functions of different subareas of a captured speckle image. Lehmann [16] carried out theoretical investigations regarding the degree of elongation in relation to the radial distance from the optical axis and the surface roughness $\mathrm{Sa}$. For $N$ different laser wavelengths, the radial speckle elongation $\Delta \xi_{s p}$ over the $\xi$-axis at $\eta=0$, normalized by the orthogonal speckle elongation $\Delta \eta_{s p}$, is

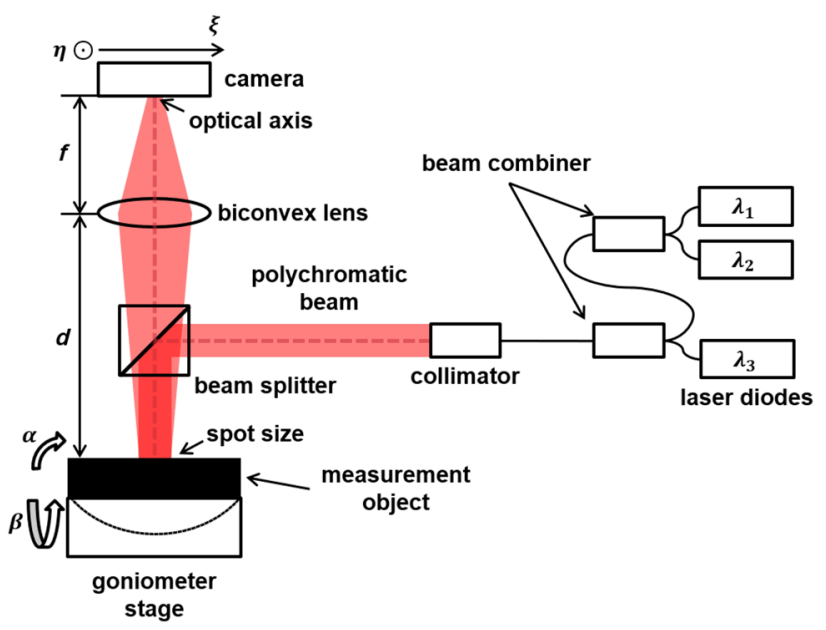

Fig. 1 Principle arrangement for roughness measurements using polychromatic speckle patterns on tilted surfaces in the trichromatic case. $f$ is the focal length of the biconvex lens, $d$ is the distance from the measurement object to the biconvex lens, $\alpha, \beta$ are the tilt angles of the measurement object and the $\lambda_{i}$ are the wavelengths for each laser diode, furthermore $\xi$ and $\eta$ are coordinates on the camera chip 


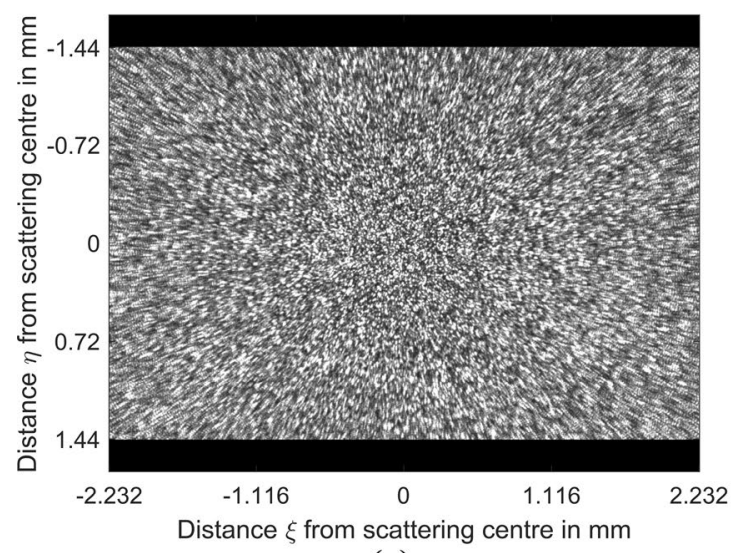

(a)

Fig. 2 a Experimentally recorded polychromatic speckle pattern (wavelengths of 633, 637, and $642 \mathrm{~nm}$ ) captured with a monochrome camera from a spark eroded surface with a roughness of $S a=0.8 \mu \mathrm{m}$. b Calculated normalized radial speckle elongation (solid lines) with

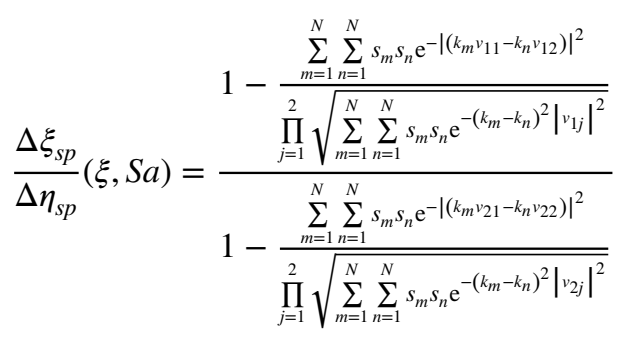

with

$v_{11}=v_{21}=v, \quad v_{12}=v+\Delta v_{2}, \quad v_{22}=v+\Delta v_{1}$

where

$v=\left(\begin{array}{c}L \xi /(2 f) \\ 0 \\ 2 S a\end{array}\right)$

and

$\Delta v_{1}=\left(\begin{array}{c}L \Delta \xi_{p x} /(2 f) \\ 0 \\ 0\end{array}\right), \quad \Delta v_{2}=\left(\begin{array}{c}0 \\ L \Delta \eta_{p x} /(2 f) \\ 0\end{array}\right)$.

The symbol $L$ denotes the $1 / \mathrm{e}^{2}$ radius of the measuring spot, $f$ the focal length of the biconvex lens (see Fig. 1), $\Delta \xi_{p x}, \Delta \eta_{p x}$ are the camera pixel size, $s_{m}, s_{n}$ the mean intensities for each wavelength and $k_{m}, k_{n}$ the wavenumbers for each wavelength.

An example of the theoretical behavior of the normalized radial elongation with respect to the radial distance $\xi$ from the scattering centre according to Eq. (2) is shown in Fig. 2b, assuming the same trichromatic illumination as in Fig. 2a and $L=0.7 \mathrm{~mm}, f=60 \mathrm{~mm}, \Delta \xi_{p x}=\Delta \eta_{p x}=6 \mu \mathrm{m}$ and $s_{m}=s_{n}=$ const. Depending on the size of the camera

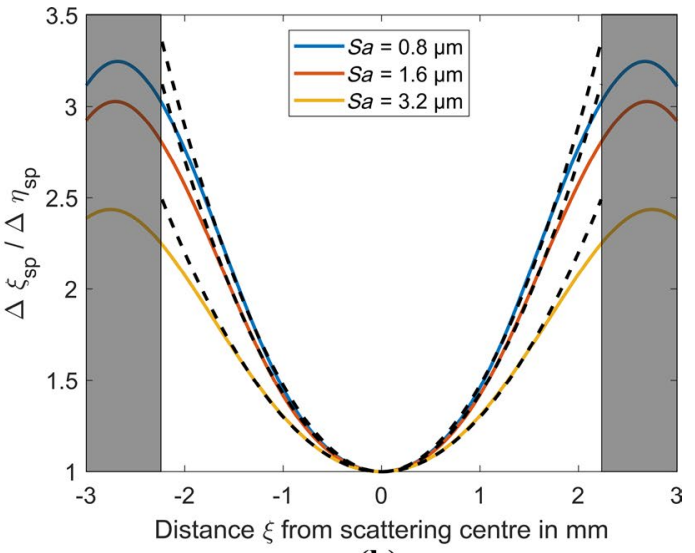

(b)

respect to the distance $\xi$ from the optical axis according to the theory from Eq. (2), considering the trichromatic illumination and different roughness values $S a$. In addition parabolic approximations (dashed lines) centred at $\xi=0$ are shown

chip, only a central cutout of the polychromatic speckle image is captured, which is illustrated by the greyed areas left and right. Beginning with a relative radial elongation $\Delta \xi_{s p} / \Delta \eta_{s p}$ at $\xi=0$ of one, it increases at first with increasing $\xi$. Around $\xi \approx 2.6 \mathrm{~mm}$ the individual speckles of the different wavelengths begin to separate so that the evaluated radial elongation then decreases and asymptotically becomes one again. The latter effect is here called speckle decorrelation. Note that a smaller wavelength difference results in a slower increase of the elongation with increasing $\xi$ as well as a shift of the radial position of the beginning decorrelation away from the scattering centre. However, this reduces the sensitivity of the measurement principle. The radial speckle elongation behavior over the radial distance obviously depends on $S a$ (see Fig. 2b) but for designing the measurement principle it is necessary to understand and to quantify this dependency.

The characteristic value of $\xi$, for which the radial elongation curves become maximal, depends mostly on the composition of the used wavelengths, the focal length $f$ of the biconvex lens and the size $L$ of the measuring spot. As a result, the position of the maximal radial elongation provides no reliable indication for the surface roughness. In order to deepen the understanding of the dependency on $S a$, a normalization of the radial speckle elongation regarding the maximum of each curve is applied (see Fig. 3). As a result, the effect of $S a$ on the curve shape in the considered interval between the two maxima around the optical axis is negligible, i.e., $S a$ mainly influences the amplitude of the radial speckle elongation curve. Therefore, a suitable determination of $S a$ would be the evaluation of the elongation curve's amplitude. To ensure that the curve maximum is captured, however, a large measurement window is required. To limit 


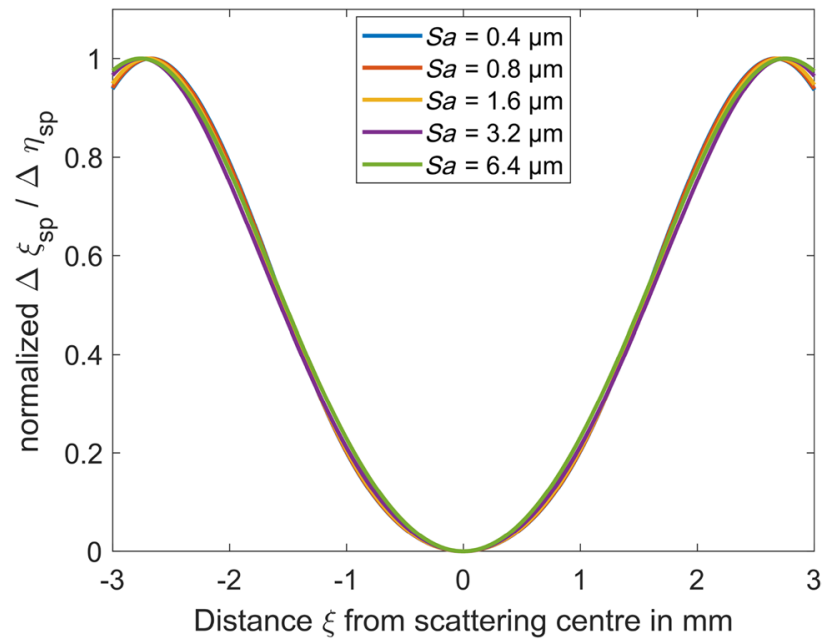

Fig. 3 Normalized theoretical curves of the radial speckle elongation with respect to the distance $\xi$ from the optical axis for different surface roughness values (trichromatic illumination, see Fig. 2)

the size of the measurement window, it is sufficient to consider the amplitude increase of the parabolic curve section near the optical axis, i.e.,

$\frac{\Delta \xi_{s p}}{\Delta \eta_{s p}}(\xi, S a) \approx \underbrace{f(S a)}_{=\Psi} \cdot \xi^{2}+1$.

As shown in Fig. $2 b$ the parabolic approximations (dashed lines) are in good agreement with the exact theory (solid lines) near the optical axis for $|\xi| \leq 1.6 \mathrm{~mm}$. Hence, the relationship between the single coefficient $\Psi$ of the parabola (3) and the roughness parameter $S a$ provides the calibration function $f$, which is shown in Fig. 4 as a solid line for the same trichromatic illumination as in Fig. 2 and for a range of $0 \mu \mathrm{m}<S a<10 \mu \mathrm{m}$. Assuming a minimal sensitivity of $|\mathrm{d} \Psi / \mathrm{d} S a|=40 / \mathrm{mm}^{3}$ as an example, a realistic measurement range for the considered trichromatic example approximately ranges is from $S a=0.7 \mu \mathrm{m}$ to $5.8 \mu \mathrm{m}$, where a linear relationship between $\Psi$ and $S a$, for $S a=0.7 \mu \mathrm{m}$ to $5.1 \mu \mathrm{m}$, see whitened interval in Fig. 4, can be assumed as,

$\Psi=f(S a)=p_{1} S a+p_{2}$

with $p_{1}, p_{2}$ as calibration curve parameters.

The roughness measurement principle, which was strictly derived from the elongation model of polychromatic speckles, is so far illustrated for conditions with no surface tilt, i.e., for the tilt angles $\alpha=0, \beta=0$. A tilting of the surface mainly results in a respective shift of the speckle pattern. The scattering centre has moved with respect to the optical axis due to the tilt to the left and an area with speckle decorrelation is captured on the right side of the image (compare Fig. 5a to Fig. 2a).

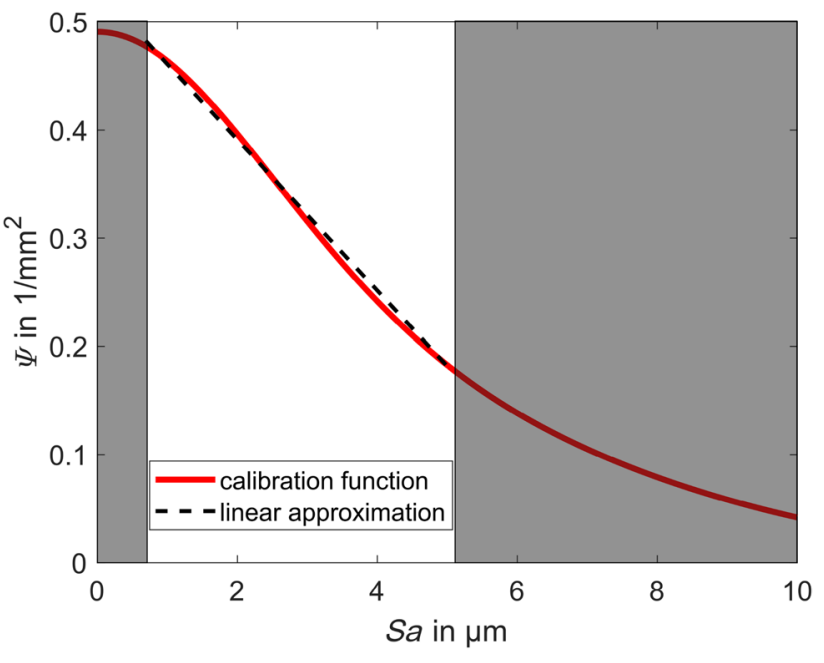

Fig. 4 Calculated calibration function from Eq. (3), which results from the relationship $\Psi=f(\mathrm{Sa})$

The distances in the image plane from the scattering centre are still denoted as $\xi, \eta$, and the shift of the scattering centre from the optical axis is introduced as $\left(\Delta \xi_{0}, \Delta \eta_{0}\right)$. The shift of the scattering centre is related to the tilt angles $\alpha$ and $\beta$ by

$\Delta \xi_{0}=d \cdot \tan \alpha$

$\Delta \eta_{0}=d \cdot \tan \beta$

where $d$ is the distance from the measurement surface to the biconvex lens (see Fig. 1). As a result, the tilt angle information can be extracted from the speckle image. On the basis of experimental observations, it is subsequently assumed as a first approximation that only the position of the observed speckle pattern changes by the tilting, so that the radial speckle elongation curves are shifted along the abscissa while the curve shape remains unchanged (see Fig. 5b). According to this hypothesis, the derived modeldriven roughness measurement principle on the basis of a parabolic fit of the radial speckle elongation enables surface roughness measurements with implicit corrections of the surface tilt. Note that the hypothesis can only be assumed for sufficiently small tilt angles, since according to Eq. (5), the shape near the minimum of the parabola that marks the scattering centre is not captured on the camera for large tilt angles. In addition to the shift in position of the parabola minimum, it also has to be considered that the tilting of the surface can cause a change in the characteristic shape of the radial speckle elongation curve, since the polychromatic speckle elongation model neglects, e.g., shadowing effects or changes in intensity. For this reason, experimental investigations are necessary to determine the maximal tilt angle for 


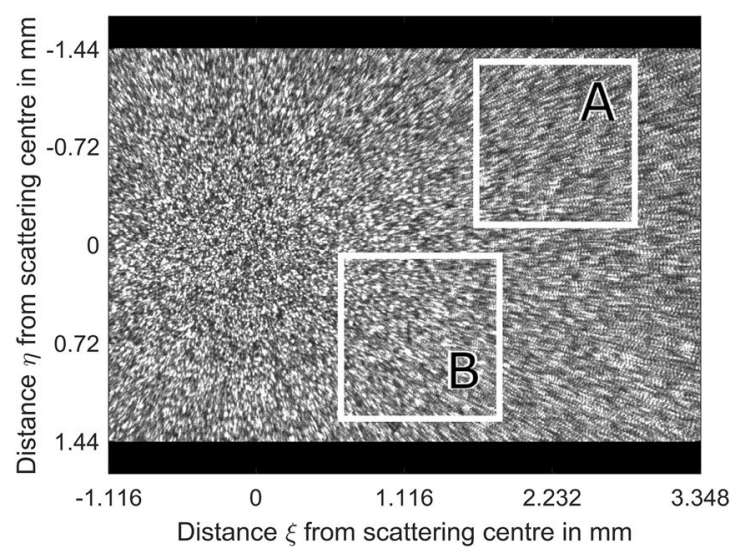

(a)

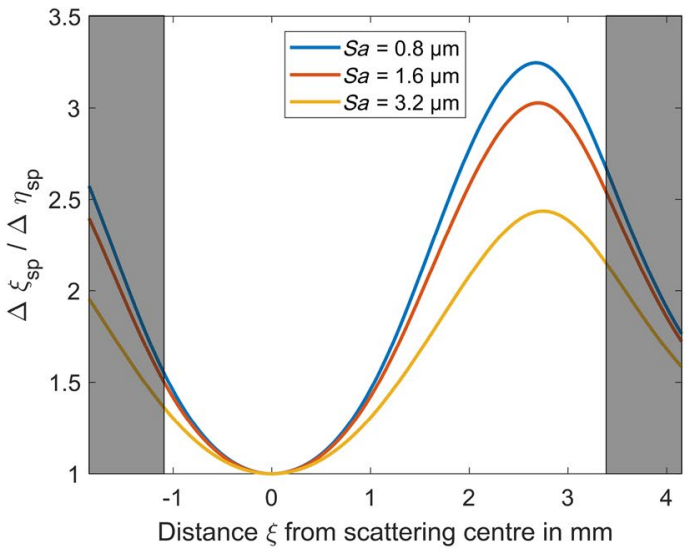

(b)
Fig. 5 a Experimentally recorded polychromatic speckle pattern (wavelengths 633, 637, and $642 \mathrm{~nm}$ ) captured with a monochrome camera obtained from a tilted, spark eroded surface with a roughness of $0.8 \mu \mathrm{m}$. The two areas A and B mark a subarea, where an autocorrelation function is used to determine the radial speckle elonga-

which the model-driven roughness measurement approach using polychromatic speckles is valid.

\section{Experimental Evaluation}

\subsection{Setup}

Three single-mode pigtailed laser diodes with the wavelengths $\lambda_{1}=633 \mathrm{~nm}, \lambda_{2}=637 \mathrm{~nm}, \lambda_{3}=642 \mathrm{~nm}$ and output powers $P_{1}=50 \mathrm{~mW}, P_{2}=50 \mathrm{~mW}, P_{3}=20 \mathrm{~mW}$ are used to illuminate the surface to be measured. A combination of the wavelengths is achieved with beam combiners with a coupling ratio of 25:25:50. The polychromatic laser beam is focused through a polarizing beam splitter onto the measuring surface. To ensure maximum light efficiency, the beam is linearly polarized by means of a polarizer in front of the beam splitter. The polarizer is adjusted so that each laser diode has a similar power after polarization. Then the deflected beam illuminates the measured surface after passing through a retardation plate, which modifies the beam state to a circular polarization. Since the light scattered from the surface passes back through the retardation plate, the polarization is changed to a linear polarization, where the direction is rotated by $90^{\circ}$ with respect to the incoming beam and therefore is not deflected by the polarizing beam splitter. The beam is then focused with a $f=60 \mathrm{~mm}$ biconvex lens onto a monochrome CCD camera (The Imaging Source, DMM 22BUC03-ML) with $744 \times 480$ pixel and a pixel size of $\Delta \xi_{p x}=\Delta \eta_{p x}=6 \mu \mathrm{m}$. The biconvex lens has a distance of $d=100 \mathrm{~mm}$ from the measurand. The measurand is a tion. b Assumed behavior of the normalized radial speckle elongation with respect to the distance $\xi$ from the scattering centre, considering the trichromatic illumination and different roughness values $S a$ for a tilted surface

roughness standard Microsurf 331 with a surface roughness of $S a=0.8 \mu \mathrm{m}, 1.6 \mu \mathrm{m}$ and $3.2 \mu \mathrm{m}$ produced by spark erosion. It is placed on two goniometer stages to allow surface inclination. The measuring spot has a size of $L=1 \mathrm{~mm}$.

\subsection{Evaluation of the Radial Speckle Elongation}

The polychromatic speckle patterns are evaluated by applying the autocorrelation function (ACF) to subareas of $240 \times 240$ pixels. In Fig. 5, as an example, two subareas are defined. A $13 \times 13$ pixels section of the vicinity around the centre of the ACF for both areas A and B is shown in Fig. 6. A radial evaluation of the speckle elongation is carried out via interpolation with a 2D-Gaussian function of the form

$$
\begin{aligned}
& \begin{aligned}
Z(j, k) & =a+b \cdot \mathrm{e}^{-\frac{n_{1}}{\Delta \xi_{s p}}{ }^{2}-\frac{n_{2}}{\Delta n_{s p}}}{ }^{2} \\
\text { with } & \\
n_{1} & =j \cdot \cos (\varphi)+k \cdot \sin (\varphi) \\
n_{2} & =j \cdot \sin (\varphi)+k \cdot \cos (\varphi),
\end{aligned}
\end{aligned}
$$

where $a$ is an offset, $b$ is the height, $\Delta \xi_{s p}$ and $\Delta \eta_{s p}$ are the radial and orthogonal speckle elongation, respectively, $j$ and $k$ are the pixel positions in the horizontal and vertical direction in the domain of $-J / 2 \leq j \leq J / 2$ and $-K / 2 \leq k \leq K / 2$ for $J=K=12$ and $\varphi$ is the orientation of the main axis of the approximated Gaussian function. According to Eq. (3), the radial elongation aspect ratio 


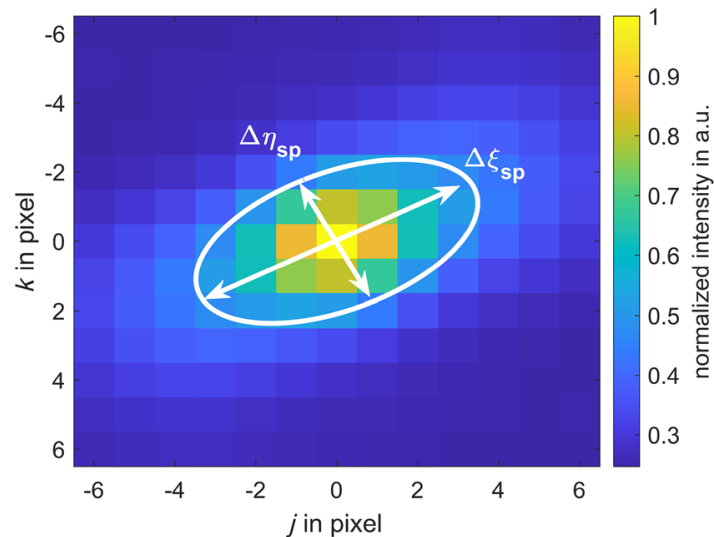

(a)

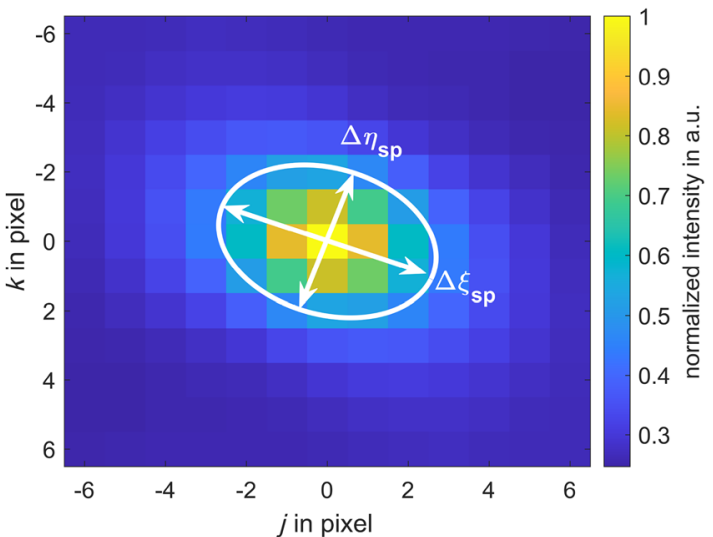

(b)

Fig. 6 Vicinity of $13 \times 13$ pixels around the centre of the ACF obtained from a Fig. 5a area A and b Fig. 5a area B. The ACF centre $(j, k)$ is at $(0,0)$

$\frac{\Delta \xi_{s p}}{\Delta \eta_{s p}} \approx \Psi \cdot r^{2}+1$

is introduced for each subarea, where $r=\sqrt{\xi^{2}+\eta^{2}}$ is the radial distance from the scattering centre. To calculate $r$, it is necessary to know the position of the scattering centre.

\subsection{Evaluation of the Scattering centre Position}

The scattering centre position is determined by the usage of the orientation angle $\varphi$ of the elongated speckle. The subarea is moved in predefined steps across the whole speckle pattern. At each position of the subarea, the autocorrelation function (ACF) is computed to determine the orientation angle $\varphi$ of the main axis. Using $\varphi$ and the centre position of the subarea, a straight line is defined, which should run through the scattering centre of the speckle pattern. The intersection of all these lines marks the best approximation of the scattering centre position in the image, see Fig. 7.

The lines are represented by a set of equations of the form

$\overrightarrow{v_{l}}=\overrightarrow{v_{1, l}}+x \overrightarrow{v_{2, l}}, \quad l=1, \ldots, M$.

$\overrightarrow{v_{1, l}}$ is the position vector, which points to the centre of the subarea. $\overrightarrow{v_{2, l}}$ is the directional vector with the orientation angle $\varphi$. The parameter $x$ is a real number and $M$ is the number of subareas. To determine the position of the scattering centre, the point with the smallest perpendicular square sum of distances to all lines is searched. Considering the distance of a point $\vec{p}(\xi, \eta)$ perpendicular to a line as

$\frac{\left|\left(\vec{p}(\xi, \eta)-\overrightarrow{v_{1,1}}\right) \times \overrightarrow{v_{2,1}}\right|}{\left|\overrightarrow{v_{2,1}}\right|}$,

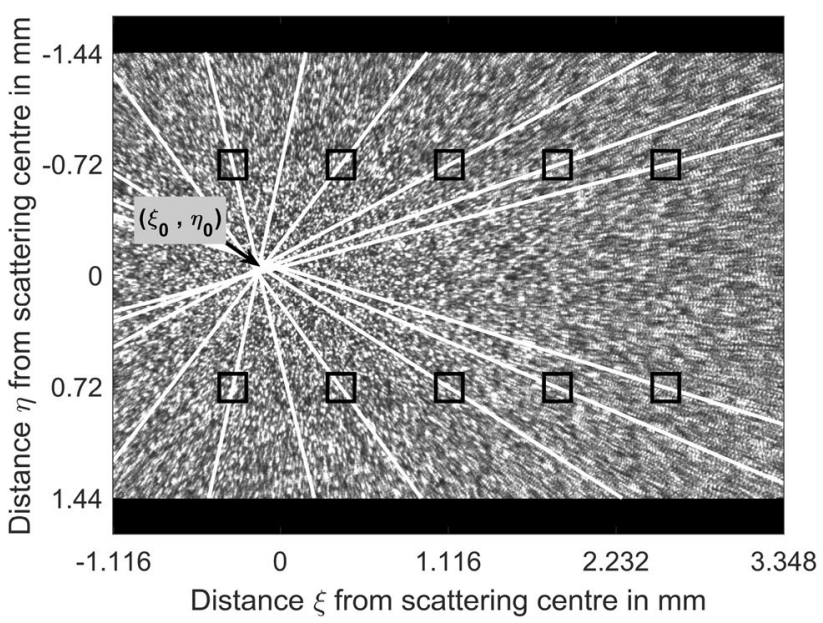

Fig. 7 Display of an intersection area by the usage of the orientation of the speckle elongation. The black-lined squares show the centre positions of some of the used subareas

the position is found by numerically solving the minimization problem

$\min _{\xi, \eta} \sum_{l=1}^{M} \frac{\left|\left(\vec{p}(\xi, \eta)-\overrightarrow{v_{1, l}}\right) \times \overrightarrow{v_{2, l}}\right|}{\left|\overrightarrow{v_{2, l}}\right|}$.

\section{Experimental Investigations}

\subsection{Calibration of the Measurement Approach}

In order to establish the relation between $\Psi$ and $S a$, several roughness values for $\alpha=0^{\circ}$ are measured using the roughness standard Microsurf 331 as well-referenced 
measurement objects. To reduce the signal-to-noise ratio, in a first approach, each captured speckle image is binarized, using the mean intensity as the threshold. Hence, only the speckle size is evaluated and the effect of the tilting intensity fluctuations is reduced. The speckle pattern is scanned by subareas of $240 \times 240$ pixels, which are moved in steps of 30 pixels until the whole speckle pattern is covered. For each subarea, the radial elongation aspect ratio $\Delta \xi_{s p} / \Delta \eta_{s p}$ and the orientation angle $\varphi$ are approximated using the model from Eq. (7). Based on these measurements, the parameters of the inverse calibration function (4) are obtained, where the mean values of $\Psi$ for the different roughness values are used for a linear approximation. A least squares fit leads to the coefficients $p_{1}=-0.1223 \mu \mathrm{m}^{-1}$ and $p_{2}=0.5337 \mathrm{~mm}^{-2}$. The resulting calibration function is shown in Fig. 8 together with the mean values and standard deviation of $\Psi$ for 100

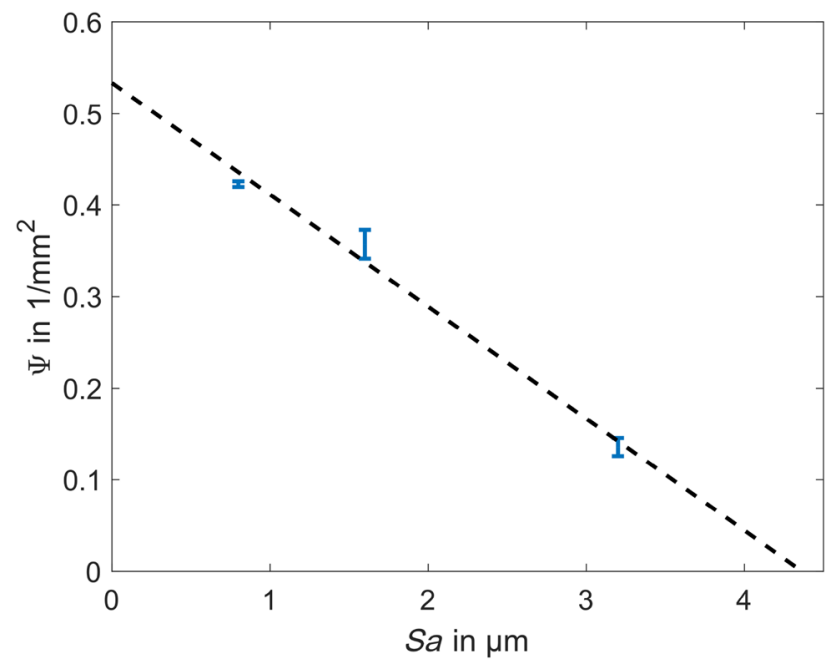

Fig. 8 Approximated calibration function (dashed line) and mean $\Psi$ (blue markers) with the standard deviation of 100 measurements for $\alpha=0^{\circ}$ measurements per roughness value. The experimentally determined calibration function shows an agreement with the theoretical considerations (see Fig. 4). Roughness measurements are finally enabled by inverting the obtained $\Psi$-Sa-relation that yields the calibration curve.

To investigate the implicit corrections of the surface tilt, the roughness standard is tilted in steps of $0.25^{\circ}$. For each tilt angle and roughness value $S a=0.8 \mu \mathrm{m}, 1.6 \mu \mathrm{m}, 3.2 \mu \mathrm{m}$, 100 pictures are taken and $\Psi$ is approximated according to Eq. (8) using radial elongation aspect ratios within a range of $|r| \leq 1.2 \mathrm{~mm}$. Three examples of the obtained elongation curves for different tilt angles $\alpha$ and roughness values $S a$ with the approximated parabola are shown in Fig. 9. The assumed parabolic shape in the vicinity of the scattering centre can be observed, even if the scattering centre lies outside of the measuring field. The measured elongation curves are therefore in good agreement with the theoretical predictions.

Based on Eq. (5), the scattering centre for tilt angle $\alpha>1.27^{\circ}$ is out of the image area of the measuring window. The roughness evaluation is therefore only performed for tilt angles smaller than $1.25^{\circ}$, i.e., where the scattering centre lies in the recorded image. The mean values of 100 $S a$-measurements each are shown in Fig. 10 over the angle of inclination $\alpha$. All associated standard deviations are in a range of less than $0.09 \mu \mathrm{m}$. Since the position of the scattering centre is estimated numerically, see Sect. 3.1, small fluctuations in the speckle pattern change the determined position. To investigate the influence of this position uncertainty on the roughness measurement, the recorded polychromatic speckle patterns are additionally evaluated with a fixed scattering centre position that is calculated according to Eqs. (5) and (6). With a fixed scattering centre position, the resulting standard deviation of the roughness measurement has the same order of magnitude as without fixing the scattering centre position. Therefore, it is concluded that the effect of an uncertain scattering centre position on the roughness measurement is negligible.

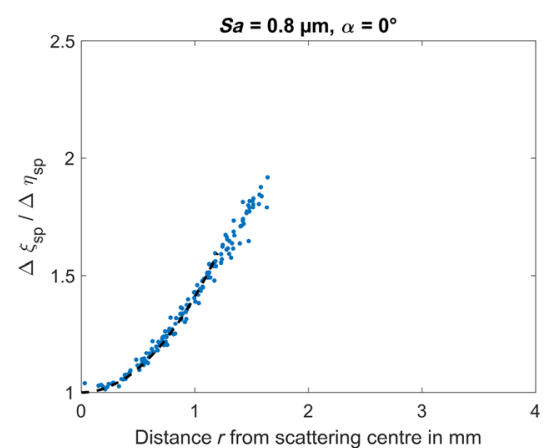

(a)

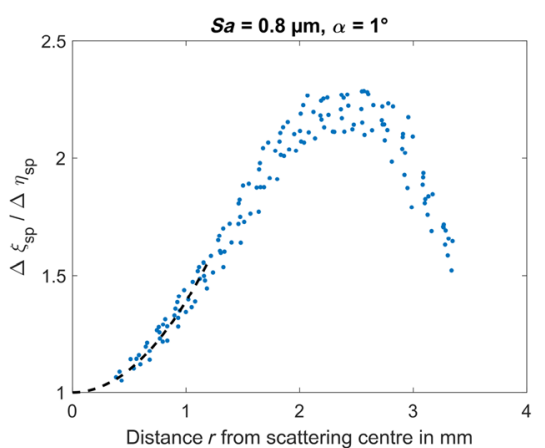

(b)

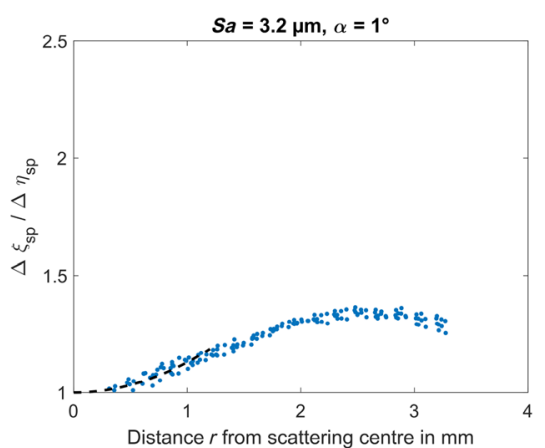

(c)

Fig. 9 Measured curves of the speckle elongation (points) with regard to the scattering centre for different roughness values and tilt angles $\alpha$ and the approximated parabola (dashed line) 


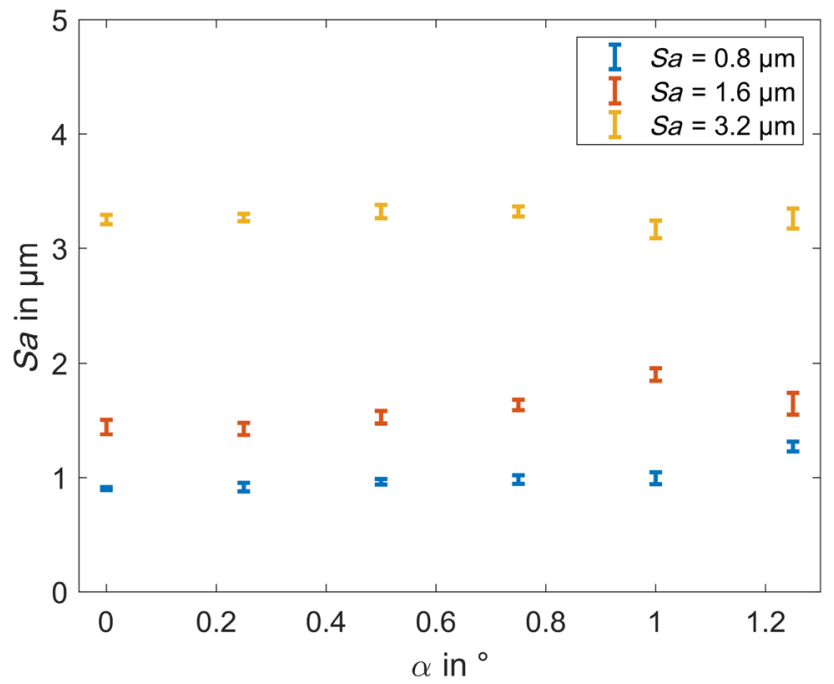

Fig. 10 Roughness evaluation algorithm for different tilt angles $\alpha$ and roughness values $S a$ with the standard deviation of 100 measurements each

According to the theoretical assumptions, the roughness parameter $S a$ is independent of the tilt angle $\alpha$. For larger tilts, slight outliers for the roughness $S a=1.6 \mu \mathrm{m}$ and $3.2 \mu \mathrm{m}$ at $\alpha=1^{\circ}$ and for $S a=0.8 \mu \mathrm{m}$ at $\alpha=1.25^{\circ}$ occur. Overall, the angle independent behavior of the measurement evaluation is confirmed, especially for small tilt angles. The maximum deviations of the mean values are smaller than $0.09 \mu \mathrm{m}$ for $S a=0.8 \mu \mathrm{m}$ and $S a=3.2 \mu \mathrm{m}$ and smaller than $0.2 \mu \mathrm{m}$ for $S a=1.6 \mu \mathrm{m}$, compared with the mean values at $\alpha=0^{\circ}$, which allows practical roughness measurements with polychromatic speckles even on tilted surfaces.

\subsection{Roughness Measurements}

In further experiments, the new evaluation approach is applied to measure the roughness from the Microsurf 331 for random tilt angles $\alpha, \beta$ within the range $\pm 1.25^{\circ}$. Each measurement is performed ten times at random positions on the surface to change the illuminated surface height distribution and thus the polychromatic speckle pattern. For comparison the roughness measurements are also evaluated using the current evaluation approach from [16] as reference, where the standard deviation of the squared speckle elongation aspect ratio over the whole polychromatic speckle pattern is used as the roughness dependent parameter. The calibration was conducted as described in Sect. 4.1. The mean values and uncertainty ranges of the roughness measurements for both evaluations are shown in Fig. 11.

The current evaluation is clearly influenced by a tilted surface, resulting in deviations from the reference value of less than $0.41 \mu \mathrm{m}$. In contrast to this, the roughness measurements with the new proposed evaluation, are in good

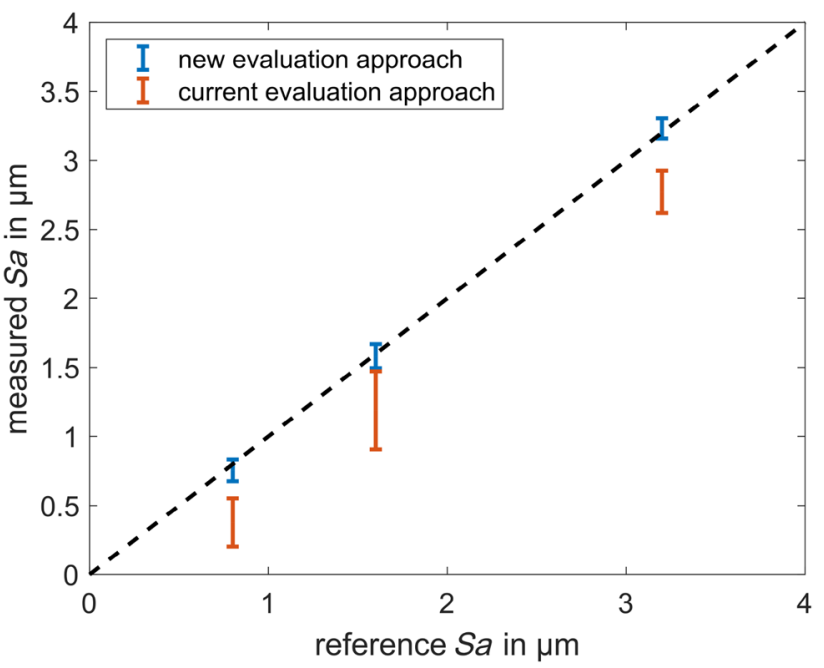

Fig. 11 Measured roughness $S a$ over the reference $S a$ for different roughness values on randomly tilted surfaces with the double standard deviation of the mean value. As a result, systematic and random errors are reduced

agreement with the corresponding reference value with negligibly small deviations of less than $0.045 \mu \mathrm{m}$. For $S a=0.8 \mu \mathrm{m}, S a=1.6 \mu \mathrm{m}$ and $S a=3.2 \mu \mathrm{m}$ the double standard deviations of the mean value are $\pm 0.078 \mu \mathrm{m}, \pm 0.087 \mu \mathrm{m}$ and $\pm 0.073 \mu \mathrm{m}$ respectively. Thus, it is shown that roughness measurements on the basis of polychromatic speckle elongation are possible within a tilt angle range of $\pm 1.25^{\circ}$ with standard deviations less than $0.1 \mu \mathrm{m}$ in a measurement range of $S a=0.8$ to $3.2 \mu \mathrm{m}$ without knowing the tilting of the surface.

\section{Conclusions and Outlook}

In this paper, an evaluation approach to compensate the influence of the tilt of the measuring surface during the polychromatic roughness measurement was presented. To reduce the tilt effects, the approach proposed here calculates the position of the scattering centre in polychromatic speckle patterns and then analyzes the roughness-dependent speckle elongation in the vicinity of and in relation to the scattering centre. Compared to the previous evaluation, without determination of the scattering centre position, the measurement uncertainty for surface roughness parameters in the range between $S a=0.8$ to $3.2 \mu \mathrm{m}$ at tilts of up to $\pm 1.25^{\circ}$ could be reduced by one order of magnitude.

Thus, fine alignment of the sensor is no longer necessary, which increases the flexibility of the measuring system and reduces the adjustment effort for varying surface alignments. Especially for in-process applications where the surface geometry is only defined within certain tolerances, the polychromatic roughness measurement system can be 
used by automatically tracking the measuring head to the target geometry.

Larger tilts still have an influence on the uncertainty of the model-based approach due to inclination-dependent intensity changes of the polychromatic speckle pattern, which have not been considered so far. In order to further reduce the measurement uncertainty and to become insensitive to larger tilt angles, non-linear calibration functions can be used in the future, which include further significant parameters such as maximum elongation. Another possibility is to compensate tilting influences by adapting the speckle pattern with a spatial light modulator [17, 18]. A further perspective for the development of the polychromatic roughness measurement method is completely new evaluation algorithms that separately detect speckle patterns of different wavelengths. With the help of the cross-correlation function, the wavelength-dependent displacement of the speckle can be evaluated without overlapping the speckle. Thus, compared to the evaluation of the polychromatic elongation of the speckle, the roughness measuring range and the insensitivity to surface tilting can be increased significantly.

Acknowledgements The project on which this article is based was funded by the Federal Ministry of Education and Research under the AiF CORNET promotion plan with the Number 232 EBG.

Funding Open Access funding enabled and organized by Projekt DEAL..

\section{Compliance with Ethical Standards}

Conflict of interest On behalf of all authors, the corresponding author states that there is no conflict of interest.

Open Access This article is licensed under a Creative Commons Attribution 4.0 International License, which permits use, sharing, adaptation, distribution and reproduction in any medium or format, as long as you give appropriate credit to the original author(s) and the source, provide a link to the Creative Commons licence, and indicate if changes were made. The images or other third party material in this article are included in the article's Creative Commons licence, unless indicated otherwise in a credit line to the material. If material is not included in the article's Creative Commons licence and your intended use is not permitted by statutory regulation or exceeds the permitted use, you will need to obtain permission directly from the copyright holder. To view a copy of this licence, visit http://creativecommons.org/licenses/by/4.0/.

\section{References}

1. Vorburger TV, Rhee HG, Renegar TB, Song JF, Zheng A (2007) Comparison of optical and stylus methods for measurement of surface texture. Int J Adv Manuf Technol 33(1-2):110

2. Biro I, Kinnell P (2020) Performance evaluation of a robotmounted interferometer for an industrial environment. Sensors 20(1):257
3. Mazule L, Liukaityte S, Eckardt R, Melninkaitis A, Balachninaite $\mathrm{O}$, Sirutkaitis V (2011) A system for measuring surface roughness by total integrated scattering. J Phys D Appl Phys 44(50):505103

4. Brodmann R, Allgauer M (1989) Surface measurement and characterization, vol 1009. International Society for Optics and Photonics, pp 111-118

5. Goodman JW (1976) Some fundamental properties of speckle. JOSA 66(11):1145

6. Kaufmann GH (2011) Advances in speckle metrology and related techniques. Wiley, Hoboken

7. Fischer A, Stöbener D (2019) In-process roughness quality inspection for metal sheet rolling. CIRP Ann 68(1):523

8. Patzelt S, Stöbener D, Fischer A (2019) Laser light source limited uncertainty of speckle-based roughness measurements. Appl Opt 58(23):6436

9. Persson U (2006) Surface roughness measurement on machined surfaces using angular speckle correlation. J Mater Process Technol 180(1-3):233

10. Salazar F, Belenguer T, García J, Ramos G (2012) On roughness measurement by angular speckle correlation. Metrol Meas Syst 19(2):373

11. Tomita Y, Nakagawa K, Asakura T (1980) Fibrous radial structure of speckle patterns in polychromatic light. Appl Opt 19(18):3211

12. Parry G (1974) Some effects of surface roughness on the appearance of speckle in polychromatic light. Opt Commun 12(1):75

13. Stansberg C (1979) Surface roughness measurements by means of polychromatic speckle patterns. Appl Opt 18(23):4051

14. Lehmann P, Patzelt S, Schöne A (1997) Surface roughness measurement by means of polychromatic speckle elongation. Appl Opt 36(10):2188

15. Lehmann $P$ (2003) In-process laser measurement methods based on Fourier analysis. Expert Verlag, Tübingen (in German)

16. Lehmann P (2002) Aspect ratio of elongated polychromatic farfield speckles of continuous and discrete spectral distribution with respect to surface roughness characterization. Appl Opt 41(10):2008

17. Bender N, Yilmaz H, Bromberg Y, Cao H (2018) Customizing speckle intensity statistics. Optica 5(5):595

18. Bender N, Yilmaz H, Bromberg Y, Cao H (2019) Introducing non-local correlations into laser speckles. Opt Express 27(5):6057

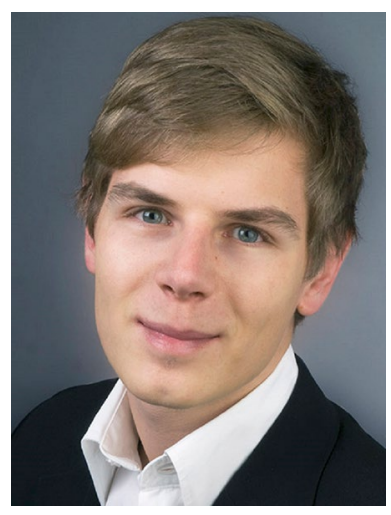

Johannes Stempin studied automation technology at the University of Clausthal, Germany. At the Bremen Institute for Metrology, Automation, and Quality Science, where he holds a position as research assistant since 2017, he works in the research group Inprocess metrology and optics. His current research focuses on model-based evaluation methods for the optical measurement of rough surfaces. 


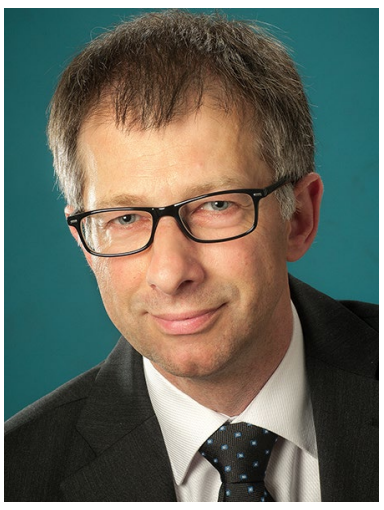

based on Maxwell equations.

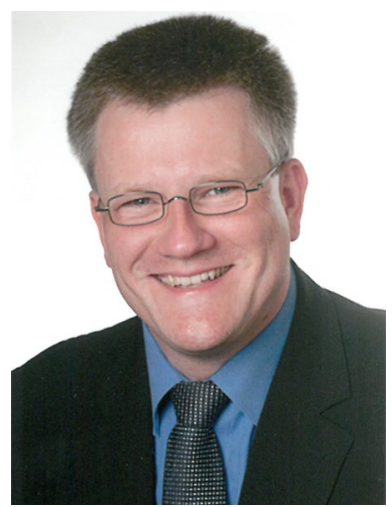

Andreas Tausendfreund studied physics at the University of Bremen and received his $\mathrm{PhD}$ in 2018 in the field of laser-optical measurement techniques for the characterization of surface defects on the nanometer scale. Since 2005 is he a research assistant at the BIMAQ and works in the research group "In-process metrology and optics". His work areas are the application of optical measurement techniques in manufacturing processes and the investigation and description of light scattering at nanostructures

Dirk Stöbener (after studying physics) received his doctorate in 2016 at the University of Bremen in the field of production engineering. Since 2003 is he the head of the research group In-process metrology and optics at BIMAQ. His research interests include acoustic and optical methods for the production related measurement of workpiece properties such as dimensions, deformation or roughness.

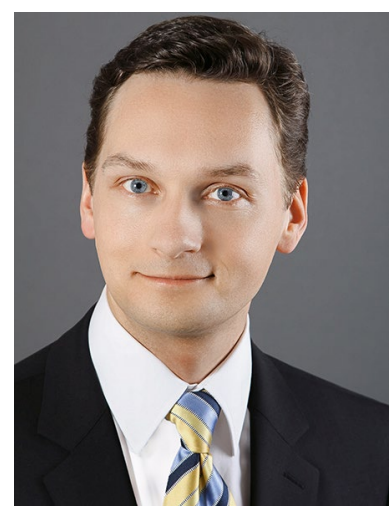

Andreas Fischer received the $\mathrm{Ph} . \mathrm{D}$. degree and the venia legendi degree in electrical engineering from Technische Universitt Dresden, Dresden, Germany, in 2009 and 2013, respectively. From 2009 to 2016, he was the head of the group of measurement system techniques at the Laboratory for Measurement and Testing Techniques of the Technische Universitt Dresden. Since 2016 he has been a Full Professor with the University of Bremen, Bremen, Germany, where he is the director of the Bremen Insti-

tute for Metrology, Automation, and Quality Science. His current research interests include the identification, characterization, and application of measurement limits as well as the development of optical measurement systems for investigating dynamic flow, shape, and surface phenomena. 